\title{
Endocrine Disrupters and Toxic Metal Ions Removal by Carboxymethyl- $\beta$-Cyclodextrin Polymer Grafted onto Magnetic Nanoadsorbents
}

\author{
Abu Zayed M. Badruddoza, Zayed Bin Zakir Shawon*, Tay Wei Jin Daniel, Kus Hidajat and \\ Mohammad Shahab Uddin \\ Department of Chemical and Biomolecular Engineering \\ National University of Singapore, Engineering Drive 4, Singapore 117576 \\ Tel: +65 65162099 , Fax: +65 67791934 \\ E-mail: shawon@nus.edu.sg
}

\begin{abstract}
In this study, carboxymethyl- $\beta$-cyclodextrin (CM- $\beta$-CD) polymer modified $\mathrm{Fe}_{3} \mathrm{O}_{4}$ magnetic nanoparticles (CMPCD$M N P S)$ is fabricated and the feasibility of using these nanoadsorbents for removal of bisphenol $A(B P A)$ and $P b^{2+}$ ions from aqueous solution was investigated. The properties of the particles were characterized by FTIR and TEM. The CM$\beta$-CD polymer grafted onto nanoparticle surface contributes to an enhancement of the adsorption capacity of $\mathrm{Fe}_{3} \mathrm{O}_{4}$ nanoparticles because of the strong complexation abilities of the multiple hydroxyl/carboxyl groups in $C M-\beta$-CD polymer with metal ions and of the hydrophobic cavity with organic contaminants through host-guest interactions. The adsorption of both BPA and $P b^{2+}$ ions onto CMPCD-MNPs was found to be dependent on $p H$. Adsorption equilibrium was achieved in 10-30 min and the adsorption kinetics of both contaminants is found to follow a pseudo-second-order kinetic model. Equilibrium adsorption data are fitted well by Langmuir isotherm model. Furthermore, these nanoadsorbents can be used as effective, separable and reusable materials for removal of both organic and inorganic contaminants from wastewater by magnetic separation systems.
\end{abstract}

\section{Introduction}

Recently, the environmental pollution such as water and soil pollutions with endocrine disrupting chemicals (EDCs) and various heavy metals has attracted global attention because of their detrimental effects on environmental and human health ${ }^{1}$. EDCs (Bisphenol A, dioxins etc.) are anthropogenic chemicals with the potential to elicit negative effects on the endocrine systems of humans and wildlife. Heavy metal ions $(\mathrm{Pb}$, $\mathrm{Cd}$, As etc.) are also toxic and non-biodegradable and hence, accumulate in human immune system and thereby disrupt function in vital organs and glands such as the heart, brain, kidneys, bone, liver, etc. Therefore, the removal of these pollutants from aquatic environment is necessary and very important.

Various treatment techniques available for the removal of these chemicals from environmental matrices are activated carbon adsorption, electrochemical, ion exchange, reverse osmosis, biological and chemical procedures $^{2}$. However, among all the methods adsorption is highly effective and economical. Recently, functionalized magnetic nanocomposites have received many attentions for use in the adsorption of both organic and inorganic pollutants ${ }^{3,4}$. Magnetic nanoadsorbents have the advantages of both magnetic separation techniques and nano-sized materials, which can be easily recovered or manipulated from complex multiphase systems with an external magnetic field. For the effective removal of toxic contaminants from wastewater, functional magnetic nanomaterials were

*Corresponding Author: Zayed Bin Zakir Shawon E-mail: shawon@nus.edu.sg synthesized by anchoring polymer, inorganic or organic molecules to the surfaces of magnetic nanoparticles including chitosan ${ }^{5}$, silica ${ }^{6}$, mesoporous carbon $^{4}$, alginate $^{3}$, gum arabic ${ }^{7}$ etc. However, no or less adsorptive study of pollutants on $\beta$-cyclodextrin $(\beta-C D)$ bonded magnetic nanoparticles has been reported, though $\beta-C D$ complexation has proved an effective method for decontaminating technique.

$\beta-C D$ is a cyclic oligosaccharide consisting of 7 glucopyranose units, which are joined together by $\alpha$ (14) linkage forming a torus-shaped ring structure with a hydrophilic exterior and a hydrophobic cavity ${ }^{8}$. It can form inclusion complexes with a wide variety of organic compounds in its hydrophobic cavity through host-guest interactions. These fascinating properties make them promising for applications in drug carrier systems, nanoreactors, bioactive supramolecular assemblies, molecular recognition, and catalysis ${ }^{9,10}$.

In this study, carboxymethyl- $\beta$-cyclodextrin polymer modified magnetic nanocomposites were synthesized and used to investigate the adsorption characteristics for removal of bisphenol $\mathrm{A}(\mathrm{BPA})$ and $\mathrm{Pb}^{2+}$ ions from aqueous solution. $C M-\beta-C D$ polymers have the ability to form inclusion complexes with organic pollutants in their hydrophobic cavities and also to adsorb metal ions due to the presence of multiple hydroxyl/carboxylic functional groups in the polymer matrix, which can interact with the metal ions by complexation reactions. The adsorption behaviors of CMPCD-MNPs with both pollutants were studied using both equilibrium and kinetic viewpoints. 


\section{Experimental}

\section{Materials}

Iron (II) chloride tetrahydrate (99\%), Iron (III) chloride hexahydrate (98\%), chloroacetic acid (99\%), Bisphenol A $(98 \%)$ was purchased from Alfa Aesar. Ammonium hydroxide (25\%) was purchased from Merck (USA). $\beta$ CD (99\%) was obtained from Tokyo Kasie Kogyo (Japan). The water in this work was Milli-Q ultrapure water.

\section{Methods}

\section{Synthesis of CM- $\beta$-CD polymer coated magnetic nanoparticles (CMPCD-MNPs)}

First, CM- $\beta$-CD polymer was prepared following the procedure as described in literature ${ }^{11}$. Briefly, $\beta-C D(5$ g) was dissolved in $50 \mathrm{ml}$ of $10 \%(\mathrm{w} / \mathrm{v}) \mathrm{NaOH}$ and 10 $\mathrm{ml}$ of epichlorohydrin were added. The system was vigorously stirred for $8 \mathrm{~h}$, another $5 \mathrm{ml}$ of epichlorohydrin added with stirring and the mixture kept overnight at room temperature. The solution was concentrated and precipitated by addition of cold ethanol $(500 \mathrm{ml})$. The gummy precipitate was then washed with ethanol and acetone and dried under high vacuum overnight. Then, two grams of the above polymer were further dissolved in $50 \mathrm{ml} 5 \%(\mathrm{w} / \mathrm{v})$ $\mathrm{NaOH}$ and $2 \mathrm{~g}$ of monochloroacetic acid were added. The system was vigorously stirred for $24 \mathrm{~h}$, neutralized with $2 \mathrm{M} \mathrm{HCl}$, concentrated to about $15 \mathrm{ml}$ and cooled to $4{ }^{\circ} \mathrm{C}$. The precipitated $\mathrm{NaCl}$ was filtered off and the supernatant was precipitated by addition of cold ethanol $(500 \mathrm{ml})$. The gummy precipitate was crushed several times with ethanol in a mortar and then washed again with ethanol and acetone and dried under high vacuum overnight.

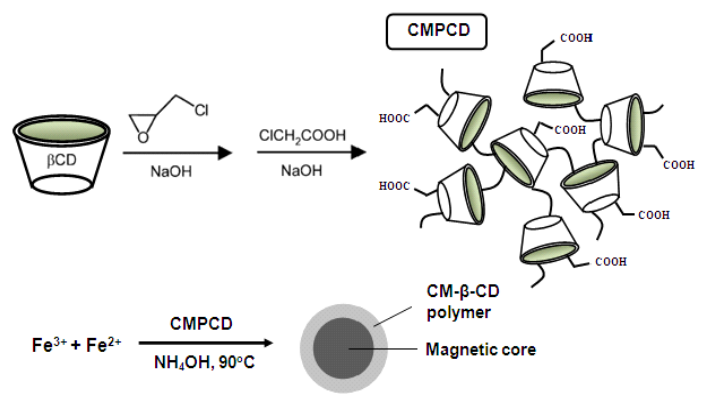

Fig.1. Scheme representation of CM- $\beta-C D$ polymer coating on bare magnetic nanoparticles.

CMPCD-MNPs were fabricated by one step coprecipitation method. Briefly, $0.86 \mathrm{~g}$ of $\mathrm{FeCl}_{2} .4 \mathrm{H}_{2} \mathrm{O}$, $2.36 \mathrm{~g} \mathrm{FeCl}_{3} \cdot 6 \mathrm{H}_{2} \mathrm{O}$ and $1.5 \mathrm{~g} \mathrm{CMPCD}$ were dissolved in $40 \mathrm{ml}$ of de-aerated Milli-Q water with vigorous stirring at a speed of $1,200 \mathrm{rpm} .5 \mathrm{ml}$ of $\mathrm{NH}_{4} \mathrm{OH}$ was added after the solution was heated to $90^{\circ} \mathrm{C}$. The reaction was continued for $1.5 \mathrm{~h}$ at $90^{\circ} \mathrm{C}$ under constant stirring and nitrogen environment. The resulting nanoparticles were then washed with Milli-Q water few times to remove any unreacted chemicals and dried in a vacuum oven.

\section{Adsorption of BPA and $\mathrm{Pb}^{2+}$ ions}

BPA and $\mathrm{Pb}^{2+}$ ions adsorption experiments were carried out using batch equilibrium technique in aqueous solutions at different $\mathrm{pH}$ range at $25^{\circ} \mathrm{C}$. In general, 120 $\mathrm{mg}$ of wet magnetic nano-adsorbent was added to 10 $\mathrm{mL}$ of BPA or $\mathrm{Pb}^{2+}$ ions solution of various concentrations and shaken in a thermostatic water-bath shaker operated at $220 \mathrm{rpm}$ for a specific period of time to reach adsorption equilibrium. The magnetic nanoadsorbents were then removed using a strong permanent magnet made of $\mathrm{Nd}-\mathrm{Fe}-\mathrm{B}$ and the supernatant was collected. The amount of BPA in the supernatant was then quantified by UV-vis spectrophotometer. The detection wavelength of BPA was set at $276.4 \mathrm{~nm}$. The concentrations of $\mathrm{Pb}^{2+}$ ions were measured using Atomic Absorption Spectrometer (Agilent ICP-MS 7700 series).

\section{Results \& Discussion}

\section{Synthesis and characterization}

The step-by-step reaction procedures to synthesize CM- $\beta-C D$ polymer modified magnetic nanoparticles are shown in Fig. 1 In this method, the carboxyl groups of $C M-\beta-C D$ polymer directly react with the surface $\mathrm{OH}$ groups on the magnetite to form Fe-carboxylate.

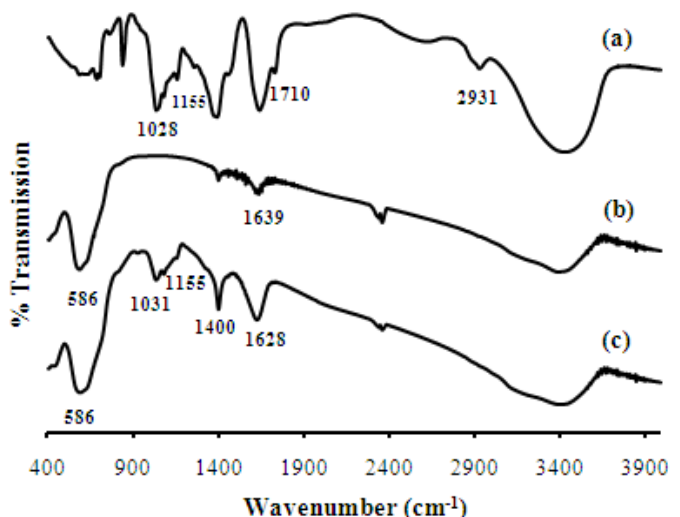

Fig.2. FTIR spectra of (a) CM- $\beta-C D$ polymer, (b) uncoated MNPs and (b) CMPCD-MNPs.

The grafting of CM- $\beta-C D$ polymer on magnetic nanoparticles is confirmed by FTIR spectroscopy. Fig. 2 shows the FTIR spectra of as-synthesized nanoparticles in the 400-4000 $\mathrm{cm}^{-1}$ wavenumber range. The spectrum of CM- $\beta$-CD polymer shows the characteristic peaks at 1028,1155 and $1710 \mathrm{~cm}^{-1}$. The peaks at 1028 and 1155 $\mathrm{cm}^{-1}$ corresponded to the antisymmetric glycosidic $v_{a}(\mathrm{C}-\mathrm{O}-\mathrm{C})$ vibrations and coupled $v(\mathrm{C}-\mathrm{C} / \mathrm{C}-\mathrm{O})$ stretch vibration. The peak at $1710 \mathrm{~cm}^{-1}$ corresponds to 
carbonyl group $(=\mathrm{CO})$ stretching which confirms the incorporation of the carboxymethyl group $\left(-\mathrm{COOCH}_{3}\right)$ into $\mathrm{CM}-\beta-\mathrm{CD}$ polymer. The characteristic adsorption band of $\mathrm{Fe}-\mathrm{O}$ bonds in the tetrahedral sites is $586 \mathrm{~cm}^{-1}$. All the significant peaks of CM- $\beta-C D$ polymer in the range of $900-1200 \mathrm{~cm}^{-1}$ are present in the spectrum of CMPCD-MNPs with a small shift. Moreover, as shown in Fig. 2(c), two main characteristic peaks appeared at 1628 and $1400 \mathrm{~cm}^{-1}$ due to bands of COOM (M represents metal ions) groups, which indicates that the $\mathrm{COOH}$ groups of $\mathrm{CM}-\beta-\mathrm{CD}$ polymer reacted with the surface $\mathrm{OH}$ groups of $\mathrm{Fe}_{3} \mathrm{O}_{4}$ particles resulting in the formation of the iron carboxylate ${ }^{12}$.

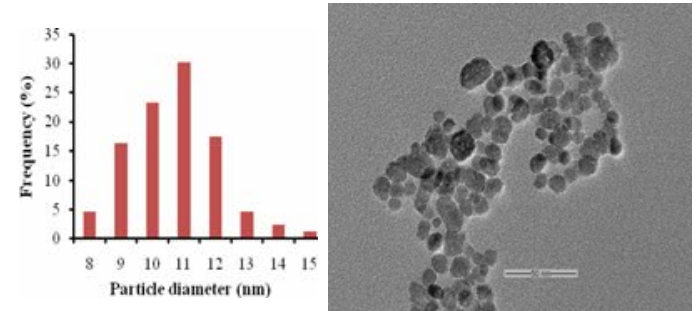

Fig.3. TEM micrograph and size distribution of CM- $\beta$ $\mathrm{CD}$ polymer coated magnetic nanoparticles. (Scale bar is $50 \mathrm{~nm}$ ).

Typical TEM image and size distribution of CMPCDMNPs are shown in Fig. 3. Well-shaped spherical or ellipsoidal magnetic nanoparticles are observed. The mean diameter of CM- $\beta-\mathrm{CD}$ coated magnetic nanoparticles is about $11 \mathrm{~nm}$.

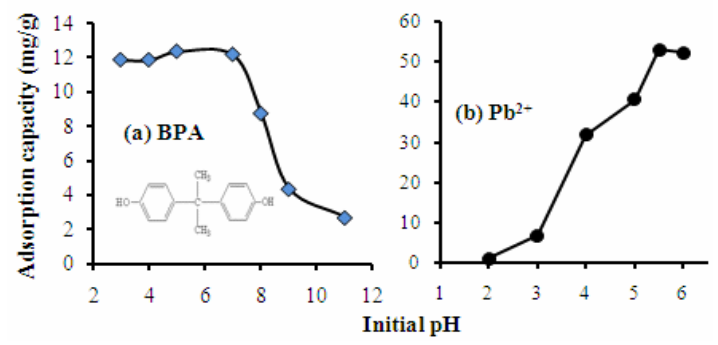

Fig.4. Effect of $\mathrm{pH}$ on the adsorption of (a) BPA and (b) $\mathrm{Pb}^{2+}$ onto CMPCD-MNPs. (BPA: $0.50 \mathrm{mg} / \mathrm{mL}$ and $\left.\mathrm{Pb}^{2+}: 200 \mathrm{mg} / \mathrm{L}\right)$.

\section{Adsorption results}

\section{Effects of pH}

The solution $\mathrm{pH}$ plays an important role in the adsorption process. The effects of initial solution $\mathrm{pH}$ on CMPCD-MNPs adsorption capacity for BPA and $\mathrm{Pb}^{2+}$ are presented in Fig. 4. It can be seen that adsorption of BPA remained roughly unchanged at $\mathrm{pH}$ below about 7.0, while increase in $\mathrm{pH}$ above about 7.0 resulted in a gradually decreased adsorption. A decreasing trend at a higher $\mathrm{pH}$ ranging from 7 to 11 was also observed when insoluble cross-linked cyclodextrin polymers were used as adsorbents ${ }^{13}$. The result could be elucidated by the $\mathrm{p} K a$ value of bisphenol-A ranging from 9.6 to $10.5^{1}$, implying that the ionization of bisphenol-A occurred at around $\mathrm{pH} 8-10$ to form the phenolate and bisphenolate anions. The phenolate anions have less tendency to be included in the hydrophobic cavity of cyclodextrin moieties, hence decrease the adsorption capacity of CMPCD-MNPs

From Fig. 4 it is also observed that the $\mathrm{Pb}^{2+}$ uptake capacity increases with an increase in $\mathrm{pH}$ from 2 to 6 . The maximum metal uptake capacity was observed at $\mathrm{pH} 5.5-6$. At $\mathrm{pH}$ below $6, \mathrm{~Pb}^{2+}$ is the major species, and with the increase of $\mathrm{pH}$ from 6 to $9, \mathrm{~Pb}(\mathrm{OH})+$ dominates $^{14}$. The variation in metal uptake capacity with $\mathrm{pH}$ can be explained by considering the differences in the charge state of metal ions, as well as the zero point of charge (ZPC) of CMPCD-MNPs $\left(\mathrm{pH}_{\mathrm{ZPC}}=4.5\right)$. Generally at $\mathrm{pH}$ below the zero point charge, $\mathrm{H}^{+}$ions compete effectively with $\mathrm{Pb}^{2+}$ ions for adsorption sites causing a decrease in uptake capacity. At $\mathrm{pH}$ above the zero point charge, the surface of the adsorbent gets negatively charged, which enhances the positively charged $\mathrm{Pb}^{2+}$ through electrostatic forces of attraction.
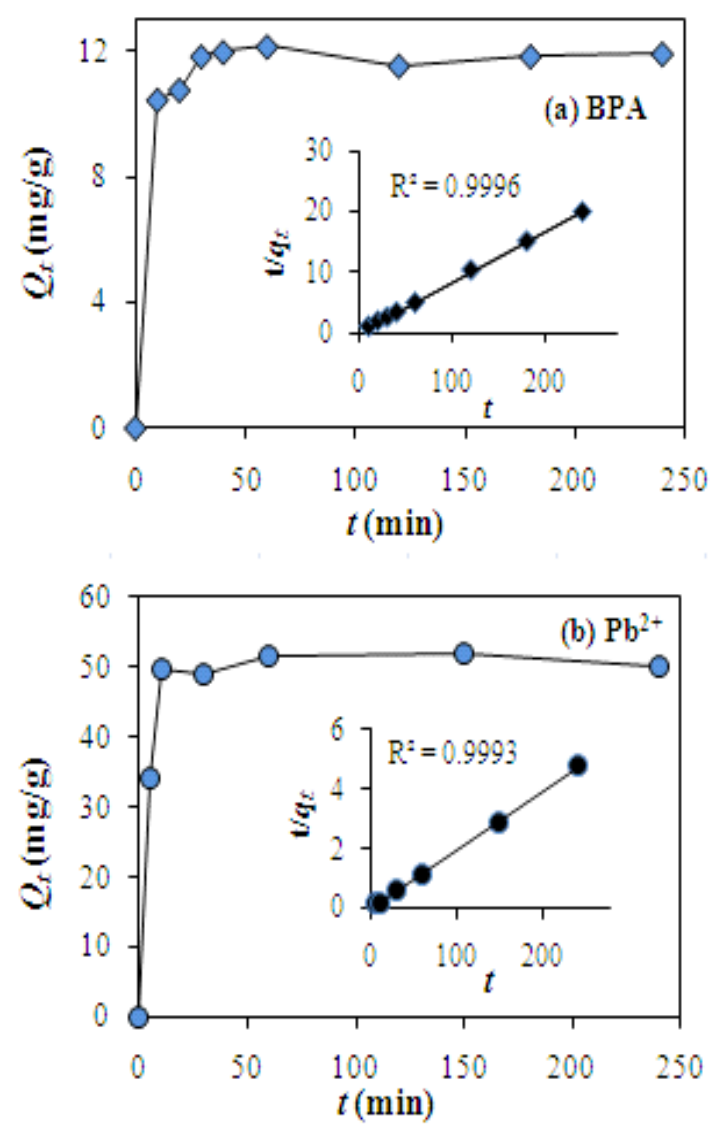

Fig.5. Effect of contact time on BPA (at $\mathrm{pH} 7$ and conc. $0.5 \mathrm{mg} / \mathrm{mL}$ ) and (b) $\mathrm{Pb}^{2+}$ adsorption by CMPCD-MNPs (at pH 5.5 and conc. $200 \mathrm{mg} / \mathrm{L}$ ). Insets: pseudo-secondorder kinetics. 


\section{Effect of contact time and kinetics}

Fig. 5 illustrates the adsorption of BPA and $\mathrm{Pb}^{2+}$ ions on CMPCD-MNPs from aqueous solution as a function of contact time. It can be seen from Fig. 5 that the maximum adsorption of both contaminants was achieved almost within 20-30 min and the adsorption remained nearly constant after that.

The pseudo-second-order rate model is often used to simulate the adsorption kinetic of contaminants on magnetic nanoadsorbents ${ }^{12}$ :

$$
\frac{t}{Q_{t}}=\frac{1}{k_{2} Q_{e}^{2}}+\frac{1}{Q_{e}} t
$$

Where $k_{2}$ is the rate constant of pseudo-second-order adsorption $\left(\mathrm{g} \mathrm{mg}^{-1} \mathrm{~min}^{-1}\right)$. The slope and intercept of the plot of $t / Q_{t}$ versus $t$ are used to calculate $k_{2}$ and $Q_{e, c a l}$ (Fig. 5). The correlation coefficient $\left(R^{2}\right)$ for the pseudosecond-order adsorption model has high value ( $>99 \%)$ which indicates that the kinetic adsorption can be well described by a pseudo-second-order rate equation. The values of $k_{2}, Q_{\mathrm{e}}$ and $R^{2}$ are $0.91 \mathrm{~g} /(\mathrm{mg} \cdot \mathrm{min}), 11.95 \mathrm{mg} / \mathrm{g}$ and 0.9996 for BPA and $0.05 \mathrm{~g} /(\mathrm{mg} \cdot \mathrm{min}), 50.76 \mathrm{mg} / \mathrm{g}$ and 0.9993 for $\mathrm{Pb}^{2+}$ ions, respectively.
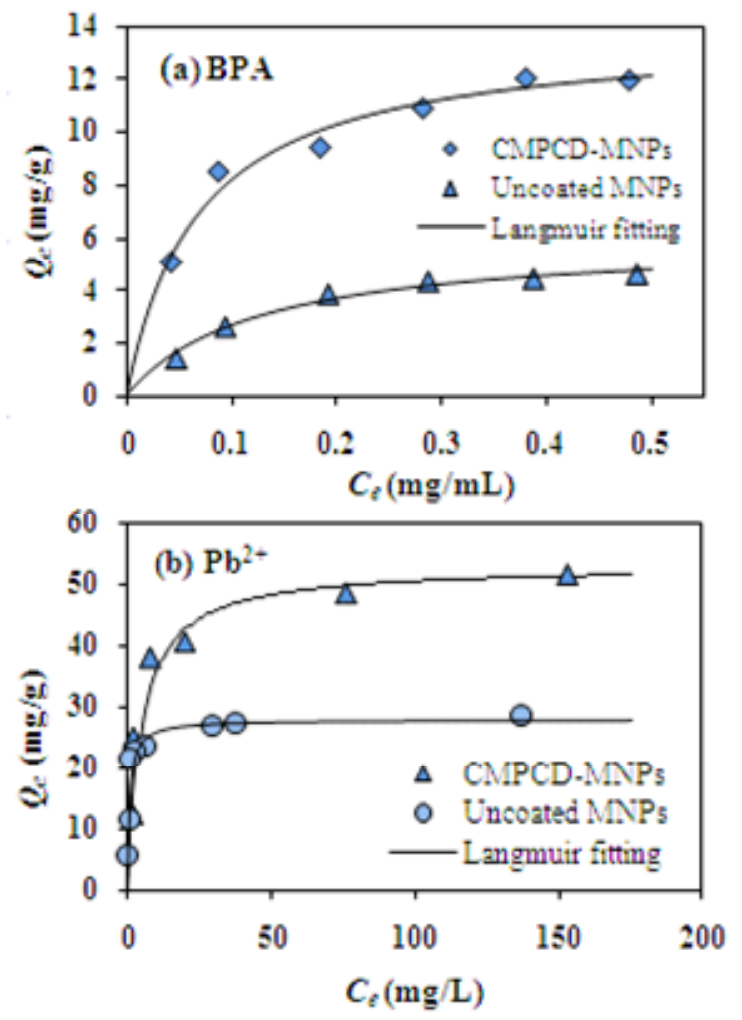

Fig.6. The adsorption isotherm of (a) BPA at pH 7 and (c) $\mathrm{Pb}^{2+}$ at $\mathrm{pH} 5.5$ onto uncoated and CMPCD-MNPS at $25^{\circ} \mathrm{C}$.

\section{Equilibrium studies}

The equilibrium isotherms for the adsorption of BPA and lead ions by uncoated MNPs and CMPCD-MNPs at $25^{\circ} \mathrm{C}$ are shown in Figuer 6 . The equilibrium data are fitted by Langmuir and Freundlich isotherm model which can be expressed in eqs 2 and 3, respectively:

$$
\begin{aligned}
& \frac{C_{e}}{Q_{e}}=\frac{C_{e}}{Q_{m}}+\frac{1}{Q_{m} K_{L}} \\
& \ln Q_{e}=(1 / n) \ln C_{e}+\ln K_{F}
\end{aligned}
$$

where $Q_{e}$ is the amount of adsorbate adsorbed per mass of adsorbent at equilibrium $(\mathrm{mg} / \mathrm{g}), C_{e}$ is the equilibrium concentration of adsorbate in aqueous solution $(\mathrm{mg} / \mathrm{mL}), Q_{\mathrm{m}}$ is the monolayer adsorption capacity at equilibrium $(\mathrm{mg} / \mathrm{g}), K_{\mathrm{L}}$ is the Langmuir equilibrium constant, $K_{F}$ is a Freundlich constant (index of adsorption capacity), $\mathrm{n}$ is Freundlich constant (index of adsorption intensity or surface heterogeneity).

Table 1. Adsorption isotherm parameters for BPA and $\mathrm{Pb}^{2+}$ ions onto uncoated and CMPCD-MNPS.

\begin{tabular}{|c|c|c|c|c|c|}
\hline \multirow{2}{*}{$\begin{array}{c}\text { Iso- } \\
\text { therm } \\
\text { Models }\end{array}$} & \multirow{2}{*}{$\begin{array}{c}\text { Para- } \\
\text { meters }\end{array}$} & \multicolumn{2}{|c|}{$\begin{array}{c}\text { Bisphenol A } \\
(\mathrm{pH} 7)\end{array}$} & \multicolumn{2}{c|}{$\begin{array}{c}\mathrm{Pb}^{2+} \\
(\mathrm{pH} 5.5)\end{array}$} \\
\cline { 3 - 6 } & $\begin{array}{c}\text { CMPCD } \\
\text { - MNPs }\end{array}$ & $\begin{array}{c}\text { Bare } \\
\text { MNP }\end{array}$ & $\begin{array}{c}\text { CMPCD } \\
\text {-MNPs }\end{array}$ & $\begin{array}{c}\text { Bare } \\
\text { MNP }\end{array}$ \\
\hline $\begin{array}{c}\text { Lang- } \\
\text { muir }\end{array}$ & $\begin{array}{c}q_{m} \\
(\mathrm{mg} / \mathrm{g})\end{array}$ & 13.70 & 5.90 & 52.20 & 28.01 \\
\cline { 2 - 6 } & $\begin{array}{c}K_{L} \\
(\mathrm{~L} / \mathrm{mg})\end{array}$ & 0.015 & 0.008 & 0.208 & 1.25 \\
\cline { 2 - 6 } & $R^{2}$ & 0.995 & 0.988 & 0.999 & 0.999 \\
\hline \multirow{2}{*}{$\begin{array}{c}\text { Freund- } \\
\text { lich }\end{array}$} & $n$ & 3.01 & 2.02 & 3.88 & 4.00 \\
\hline & $\begin{array}{c}K_{F} \\
(\mathrm{~L} / \mathrm{g})\end{array}$ & 0.016 & 0.008 & 16.43 & 13.46 \\
\hline & $R^{2}$ & 0.919 & 0.919 & 0.853 & 0.731 \\
\hline
\end{tabular}

The isotherm parameters and related $R^{2}$ values are shown in Table 1 . The adsorption isotherm data of both contaminants on this adsorbent are better fitted to Langmuir isotherm model $\left(R^{2}>0.99\right)$ in compared to Freundlich model. Based on Langmuir isotherms, the maximum adsorption capacities $\left(Q_{m}\right)$ of CMPCD-MNPs toward $\mathrm{BPA}$ and $\mathrm{Pb}^{2+}$ are 13.70 and $50.5 \mathrm{mg} / \mathrm{g}$, respectively at $25^{\circ} \mathrm{C}$, which are almost or more than twice than those obtained using uncoated MNPs. These results indicate that the modification of magnetite surface by $C M-\beta-C D$ polymer could enhance the adsorption capabilities of CMPCD-MNPs.

The inner cores of CM- $\beta-C D$ molecules, with their hydrophobic cavities, easily adsorb BPA through hostguest interactions. The main interactions in the complexation process between cyclodextrin and organic molecule are dipole-dipole, hydrogen bonding, van der 
Waals, hydrophobic and charge transfer interaction ${ }^{8}$. On the other hand, the multiple oxygen containing groups (mainly, hydroxyl/carboxyl groups) present in CM- $\beta$ $\mathrm{CD}$ polymer can form strong complexes with $\mathrm{Pb}^{2+}$ ions on the surface of CMPCD-MNPs ${ }^{12}$.

\section{Conclusion}

In summary, $C M-\beta-C D$ polymer modified magnetic nanoparticles with magnetic and inclusion/complexation properties were successfully synthesized. The adsorption of BPA and $\mathrm{Pb}^{2+}$ ions on these adsorbents was found to be $\mathrm{pH}$ dependant. The kinetics of both pollutants onto CMPCD-MNPs followed the pseudo-second-order model. The equilibrium data were fitted well by the Langmuir isotherm model. The grafted CM- $\beta-C D$ polymer contributed to enhanced adsorption capacity of CMPCD-MNPs for both BPA and $\mathrm{Pb}^{2+}$ ions from aqueous solution. Consequently, the as-obtained magnetic-cyclodextrin nanocomposites can be used as a reusable absorbent for fast, convenient, and highly efficient removal of both organic and inorganic toxic pollutants from the wastewater.

\section{References}

1. Hua, W. S., Zhi, D. B., and Yu, H. (2010). Adsorption of bisphenol A by polysulphone membrane. Desalination. 253: 22-29.

2. Gupta, V. K., and Suhas. (2009). Application of low-cost adsorbents for dye removal - A review. J. Env. Management, 90(8): 2313-2342.

3. Rocher, V., Siaugue, J. M., Cabuil, V., and Bee, A. (2008). Removal of organic dyes by magnetic alginate beads. Water Res. 42: 1290 - 1298.

4. Zhang, Y., Xu, S., Luo, Y., Pan, S., Ding, H., and Li, G. (2011). Synthesis of mesoporous carbon capsules encapsulated with magnetite nanoparticles and their application in wastewater treatment. J. Mater. Chem. 21: 3664-3671.
5. Zhou, Y. T., Nie, H. L., White, C. B., He, Z. Y., and Zhu, L. M. (2009). Removal of $\mathrm{Cu}^{2+}$ from aqueous solution by chitosan-coated magnetic nanoparticles modified with $\alpha$-ketoglutaric acid. J. Colloid Interface Sci. 330: 29-37.

6. Girginova, P. I., Silva, D., Lopes, A. L., Figueira, C. B., Otero, P., Amaral, M., Pereira, V.S., and Trindade, E. T. (2010). Silica coated magnetite particles for magnetic removal of $\mathrm{Hg}^{2+}$ from water. J. Colloid and Interface Sci. 345: 234-240.

7. Banerjee, S. S., and Chen, D. H. (2007). Fast removal of copper ions by gum arabic modified magnetic nano-adsorbent. J. Haz. Mat. 147(3): 792799.

8. Szejtli, J. (1998). Introduction and general overview of cyclodextrin chemistry. Chem. Rev. 98: 743-1754.

9. Connors, K. A. (1997). Chem. Rev. 97: 13251357.

10. Uekama, K., Hirayama F. and Irie, T. (1998). Chem. Rev. 98: 2045-2076.

11. Fernández, M., Villalonga, M. L., Fragoso, A., Caob, Baños, Maysa, Villalonga, and Reynaldo. (2004). $\alpha$-Chymotrypsin stabilization by chemical conjugation with O-carboxymethyl-poly- $\beta$ cyclodextrin. Process Biochemistry. 39: 535-539.

12. Badruddoza, A. Z. M., Tay, A. S. H., Tan, P. Y., Hidajat, K., and Uddin, M. S. (2011). Carboxymethyl- $\beta$-cyclodextrin conjugated magnetic nanoparticles as nano-adsorbents for removal of copper ions: Synthesis and adsorption studies. J Haz Mat. 185: 1177-1186.

13. Kitaoka, M., and Hayashi, K. (2002). Adsorption of Bisphenol A by Cross-Linked $\beta$-Cyclodextrin Polymer. J Inc. Phen. Macrocyclic Chem. 44: 429431.

14. Jin, L., and Bai, R. (2002). Mechanisms of Lead Adsorption on Chitosan/PVA Hydrogel Beads. Langmuir. 18: 9765-9770. 